Research article

\title{
Experimental manipulation of radiographic density in mouse mammary gland
}

\author{
Mehrdad Hariri', Geoffrey A Wood ${ }^{1}$, Marco A DiGrappa ${ }^{1}$, Michelle MacPherson², \\ Stephanie A Backman'1, Martin J Yaffe ${ }^{2}$, Tak W Mak ${ }^{1}$, Norman F Boyd ${ }^{1}$ and Rama Khokha ${ }^{1}$
}

1Department of Medical Biophysics, Ontario Cancer Institute/University Health Network, Toronto, Ontario, Canada
2Imaging Research, Sunnybrook and Women's Health Sciences Centre, Toronto, Ontario, Canada

Corresponding author: Rama Khokha, rkhokha@uhnres.utoronto.ca

Received: 4 Feb 2004 Revisions requested: 10 Mar 2004 Revisions received: 12 May 2004 Accepted: 1 Jun 2004 Published: 9 Jul 2004

Breast Cancer Res 2004, 6:R540-R545 (DOI 10.1186/bcr901)

(c) 2004 Hariri et al.; licensee BioMed Central Ltd. This is an Open Access article: verbatim copying and redistribution of this article are permitted in all media for any purpose, provided this notice is preserved along with the article's original URL.

\begin{abstract}
Introduction Extensive mammographic density in women is associated with increased risk for breast cancer. Mouse models provide a powerful approach to the study of human diseases, but there is currently no model that is suited to the study of mammographic density.
\end{abstract}

Methods We performed individual manipulations of the stromal, epithelial and matrix components of the mouse mammary gland and examined the alterations using in vivo and ex vivo radiology, whole mount staining and histology.

Results Areas of density were generated that resembled densities in mammographic images of the human breast, and the nature of the imposed changes was confirmed at the cellular level. Furthermore, two genetic models, one deficient in epithelial structure (Pten conditional tissue specific knockout) and one with hyperplastic epithelium and mammary tumors (MMTV-PyMT), were used to examine radiographic density.

Conclusion Our data show the feasibility of altering and imaging mouse mammary gland radiographic density by experimental and genetic means, providing the first step toward modelling the biological processes that are responsible for mammographic density in the mouse.

Keywords: breast cancer, mammographic density, mammography, mouse model, radiography

\section{Introduction}

The radiological appearance of the breast on mammography varies greatly among women because of the different $\mathrm{X}$-ray attenuation characteristics of fat, and epithelial and stromal components that comprise the breast tissue [1,2]. A method of classifying these variations was described by Wolfe in 1976 [3], and extensive mammographic density is now recognized to be one of the most important risk factors for human breast cancer [4-13]. Women with mammographic density in more than $75 \%$ of breast tissue are at four to six times higher risk for developing breast cancer than those with little or no density $[5,10,14]$. Although the elements that constitute mammographic density are poorly understood at present, there is evidence that stromal and epithelial cells, as well as collagen, contribute toward density in human mammographic images [15-17].

Disease modelling in mice has become a powerful tool for investigating the physiological and genetic factors that are involved in human disease. It is not known whether mammary gland density is a risk factor in mice because a suitable mouse model in which to study mammary density is currently lacking. As a necessary first step toward the eventual goal of developing such a mouse model, here we asked whether mammographic density could be altered within the mouse mammary gland. In particular, we designed our study to evaluate whether experimental manipulation of individual compartments, namely the stromal cells, matrix, or epithelium, leads to altered radiological mammographic density. We also took a genetic approach to address the correlation between epithelial structure and mammographic density using genetically engineered mouse mutants.

\section{Methods}

The fourth inguinal mammary glands were selected for the study because they contain a centrally located lymph node that can guide the site of inoculation, and because they 
have been widely studied [18-20]. Injections were always performed frontal to the lymph node. The contralateral inguinal mammary gland in each mouse was used as the sham control. Inoculations included a $40 \mu$ volume of either Matrigel (Becton Dickinson, Mississauga, Ontario, Canada) or cell media containing $5 \times 10^{6}$ Swiss 3T3 fibroblasts. Mammary glands following experimental manipulations or sham (surgery followed by the needle insertion) were isolated 5 days later. For ex vivo imaging studies, each gland was successively subjected to the following regimen: immediate radiography using Faxitron Specimen Radiography System (Model MX-20; 5 s exposure time at $18 \mathrm{kV}$; Faxitron X-Ray Corp., Wheeling, Illinois, USA) on the tissue placed in a plastic petri dish; whole mount carmine alum staining upon mounting the tissue on a glass slide using standard protocols [19]; and histology following tissue fixation and paraffin embedding.

For in vivo imaging studies, 5 days after experimental manipulations $x$-ray images of anaesthetized mice were taken using Faxitron Specimen Radiography System (Model MX-20; $10 \mathrm{~s}$ exposure time at $24 \mathrm{kV}$ ). The uninjected contralateral glands served as controls. Mammary glands were positioned flat using $25 \mathrm{G}$ needles. Mammary glands were then Isolated and imaged ex vivo.

All purchased mice were females from The Jackson Laboratory (Bar Harbor, Maine, USA). Strains used were: a) FVB/N, and b) BALB/c Foxn1nu/Foxn1nu nude mice, c) MMTV-PyMT transgenic on FVB/N background, and d) Pten-deficient mice were in a mixed background of C57BL/ 6 and 129 SvEvTac strain, in these mice inactivation of Pten has been achieved in several organs including breast using the Cre-loxP system (Pten/oxP/loxP; Mmtv-cre mice) [21,22]. The epithelial ductal tree is severely stunted in these mice and serves as a model of epithelial hypoplasia. The animals were cared for in accordance with the guidelines established by the Canadian Council on Animal Care.

\section{Results and discussion}

As illustrated, sham treated mammary glands produced a homogeneous radiological image (Fig. 1a), and the mammary whole mount (Fig. 1b) indicated little intragland variation. Histology showed an epithelial ductal tree emanating from the teat region (arrowhead), spanning the mammary fat pad (Fig. 1c) and embedded within the adipose compartment (arrowheads in Fig. 1d). Trichrome-stained tissue (Fig. 1e) showed ductal epithelium composed of a single layer of epithelial cells (arrowhead) surrounded by stromal fibroblasts (arrow) that lay within the extracellular matrix (blue staining). Next, we undertook to alter these specific components of the mammary tissue.

Matrigel, which is basement membrane matrix derived from Engelbreth-Holm-Swarm mouse sarcoma, and is com- posed mostly of laminin, collagen type IV, heparan sulphate proteoglycans, entactin and nidogen, was administered to produce local matrix deposition. When examined 5 days later by radiology, the Matrigel injected site was evident as a pronounced radiologically dense area (arrowheads in Fig. 1f), which was also apparent in the whole mount (arrowheads in Fig. 1g). Trichrome staining of mammary tissue sections confirmed the presence of collagens embedded within the mammary fat pad at this particular location (arrowheads in Fig. 1h). Higher magnification images showed collagen surrounding clusters of cells (Fig. 1i,1j).

Next, we reasoned that fibroblasts would provide a means of increasing the stromal cell component of the mammary gland. Figure $1 \mathrm{k}$ shows the radiological image following an inoculation of Swiss 3T3 fibroblasts. A highly dense pocket was evident in the mammographic radiograph (arrowheads), and a cellular mass could be seen frontal to the lymph node in the whole mount (Fig. 1l) and histology (Fig. $1 \mathrm{~m}$ ), as highlighted by the arrowheads. Fibroblasts (arrowheads) were the major cellular component of this mass, with some infiltration by polymorphonuclear cells (arrows) that probably represented a limited inflammatory response (Fig. 1n,10).

Finally, for manipulation of the epithelial compartment, mammary glands were taken from pregnant mice at day 13 of gestation. During pregnancy, alveolar epithelial cells proliferate and differentiate to become secretory alveoli in preparation for lactation, replacing much of the adipose compartment such that the mammary gland is primarily comprised of epithelial structures [23-25]. Radiology revealed a smattering of density dispersed throughout the gland, with most densities nearest to the lymph node, and gradually fading toward the edges of the gland (Fig. 1p). This density mirrored the epithelial ductal tree at the whole mount level (Fig. 1q), and an abundance of lobuloalveoli was evident at the cellular level (Fig. $1 \mathrm{r}, 1 \mathrm{~s}, 1 \mathrm{t}$ ). All of the above observations were reproduced in at least three independent experiments.

Having found that expanded epithelial structures formed during gestation correlated with increased mammographic density, we next examined whether mammary gland with hypoplastic epithelium would exhibit reduced radiographic density. Seven-week-old Pten conditional knockout mice, which have severely attenuated ductal epithelium (Fig. $2 \mathrm{f}, 2 \mathrm{~g}, 2 \mathrm{~h}, 2 \mathrm{i}, 2 \mathrm{j})$, and their age-matched wild-type littermates (Fig. 2a,2b,2c,2d,2e) were examined. At this age, the epithelial ductal tree typically grows past the lymph node in wild-type mice, covering two-thirds of the mammary fat pad (Fig. 2b). A reduction in density was noticeable in the mouse mammographic images of Pten-deficient mice as compared with control animals (Fig. $2 f$ versus Fig. 2a). Whole mounts (Fig. $2 \mathrm{~g}$ versus Fig. 2b) and histology (Fig. 
Figure 1
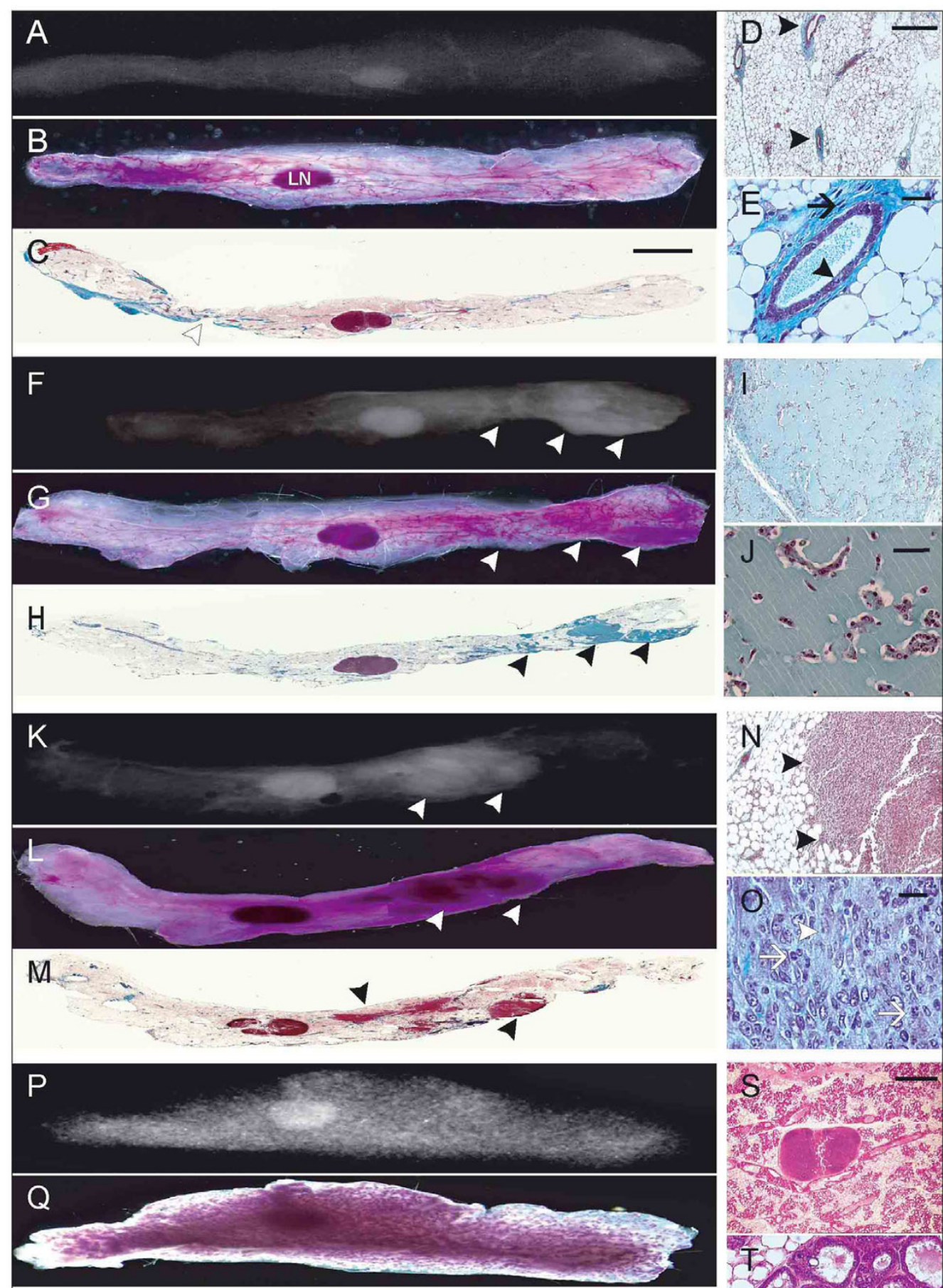

$\mathrm{R}$

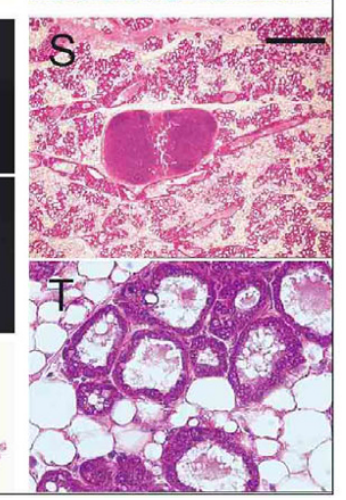

Generation of mammographic densities in the mouse mammary gland. Panels a-e represent a mammary gland from sham treated mice, $\mathbf{f}-\mathbf{j}$ illustrate Matrigel injected mammary glands, $\mathbf{k}-\mathbf{o}$ show fibroblast inoculated mammary gland, and $\mathbf{p}-\mathbf{t}$ show a mammary gland from a pregnant mouse at day 13 of gestation. Each set of glands was successively analyzed by radiography (panels $a, f, k$ and $p$ ), carmine alum whole mount staining (panels $b, g$, $\mathrm{I}$ and $\mathrm{q}$ ), and trichrome staining (panels $\mathrm{c}-\mathrm{e}, \mathrm{h}-\mathrm{j}$ and $\mathrm{m}-\mathrm{o}$ ) or haematoxylin and eosin staining (panels $\mathrm{r}-\mathrm{t}$ ). The size bar in panel $\mathrm{c}$ is $2 \mathrm{~mm}$, and this magnification also applies to panels $\mathrm{a}, \mathrm{b}, \mathrm{f}-\mathrm{h}, \mathrm{k}-\mathrm{m}$ and $\mathrm{p}-\mathrm{r}$. The size bar in panel $\mathrm{d}$ is $0.2 \mathrm{~mm}$ (which also applies to panels $\mathrm{i}$ and $\mathrm{n}$ ); in panel e it is 20 $\mu \mathrm{m}$; in panel $\mathrm{j}$ it is $0.04 \mathrm{~mm}$ (which also applies to panel $\mathrm{t}$ ); in panel o it is $10 \mu \mathrm{m}$; and in panel $\mathrm{s}$ it is $1 \mathrm{~mm}$. LN, lymph node. 
Figure 2

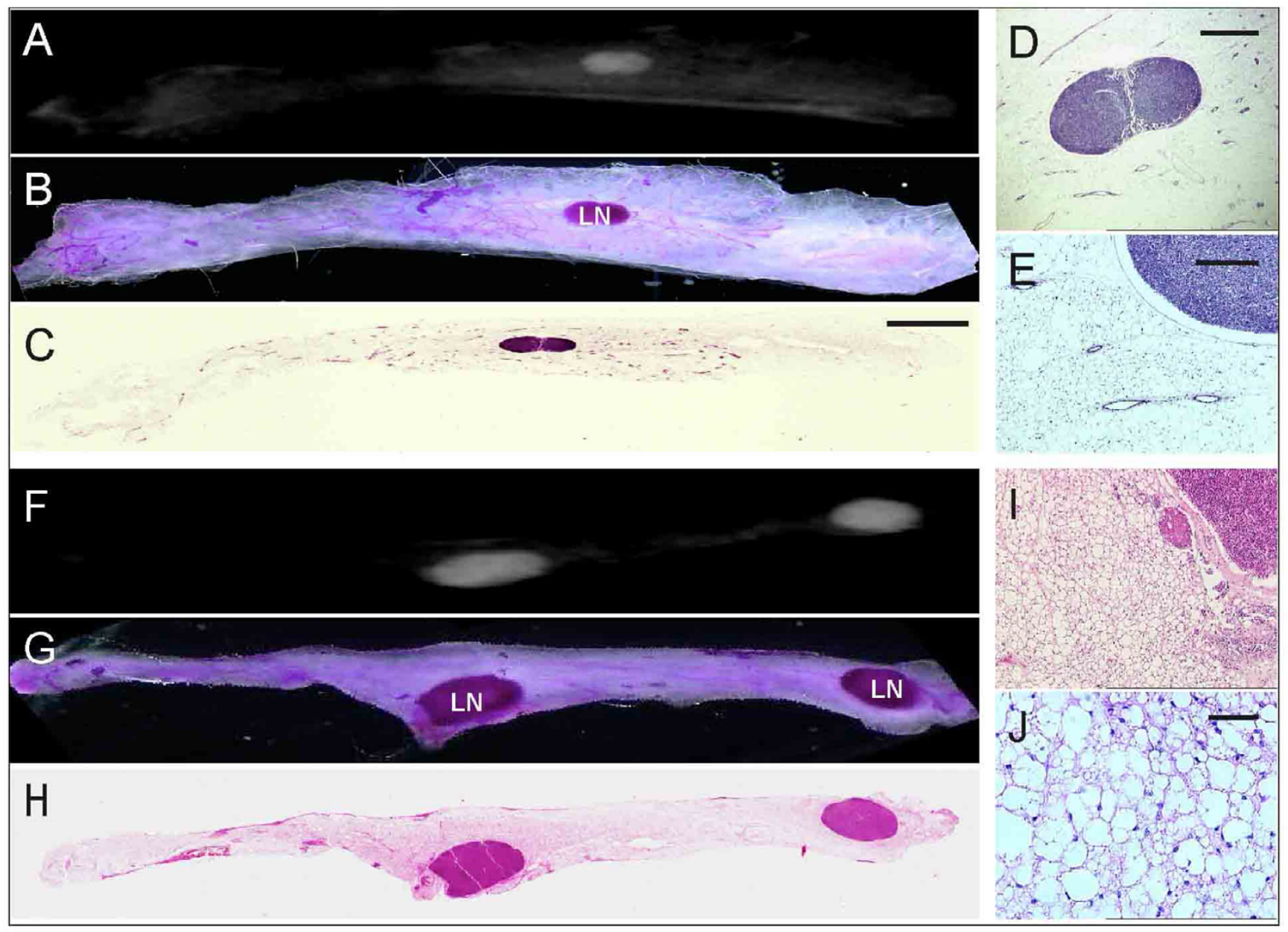

Mouse mammographic images of Pten ${ }^{\text {oxP } P / l o x P} ;$ Mmtv-cre mice. (a-e) Wild-type littermates or $(\mathbf{f}-\mathbf{j})$ mammary gland-specific pten deficient mice were successively subjected to radiography (panels a and f), carmine alum whole mount staining (panels $b$ and $g$ ) and haematoxylin and eosin staining (panels $\mathrm{c}$ and $\mathrm{h}$ ). Higher magnification of the mammary tissue (panels $\mathrm{d}, \mathrm{e}, \mathrm{I}$ and $\mathrm{j}$ ) are shown. The size bar in panel $\mathrm{c}$ is $2 \mathrm{~mm}$, and this magnification also applies to panels $a, b$ and $f-h$. The size bar in panel $d$ is $1 \mathrm{~mm}$; in panel $\mathrm{e}$ it is $0.2 \mathrm{~mm}$ (which also applies to panel i); and in panel $\mathrm{j}$ it is $0.04 \mathrm{~mm}$. LN, lymph node.

$2 \mathrm{~h}$ versus Fig. 2c) of Pten-deficient mice exhibited an absence of epithelial and stromal structures. Lymphomas are prevalent in these mutant animals by 6 weeks of age [22], and are noticeable in Fig. 2.

Next we generated and imaged mammographic density in intact mammary glands in vivo. In order to avoid any potential interference from the hair coat on radiographic imaging, we chose to use nude mice in our initial in vivo experiments. Radiographic imaging was performed 5 days after injection of either matrigel or fibroblasts, or at day 14 of gestation (Fig. 3c,3d,3e,3f,3g,3h). Mice were anaesthetized, and the fourth mammary gland was flattened by pinning. Figure $3 a$ and $3 \mathrm{~b}$ show a representative unmanipulated mammary gland with homogeneous radiographic density. The margins of the mammary gland are demarcated by yellow dotted lines, and the lymph nodes are indicated. Injected cells and matrigel created a region of density (arrowheads) distinguishable from the surrounding normal gland (Fig. 3c,3f). Such regions were not present in any of the untreated contralateral glands, which served as controls for each mouse (Fig. 3a,3b). Increased density was also apparent in the mammary gland of a mouse at day 14 of gestation, but was less well demarcated because of its diffuse nature (Fig. $3 g, 3 h)$. Ex vivo radiography of all of these glands confirmed the presence of density observed in vivo (Fig. $3 b, 3 d, 3 f, 3 h, 3 j)$.

Using a well established mammary tumor model, the MMTV-PyMT mouse, we next imaged the hyperplastic and tumor bearing mammary gland by radiography in an intact 80-day-old mouse. Figure $3 \mathrm{i}$ and $3 \mathrm{j}$ illustrate the highly increased in vivo and ex vivo radiographic density that results from mammary tumor formation. 

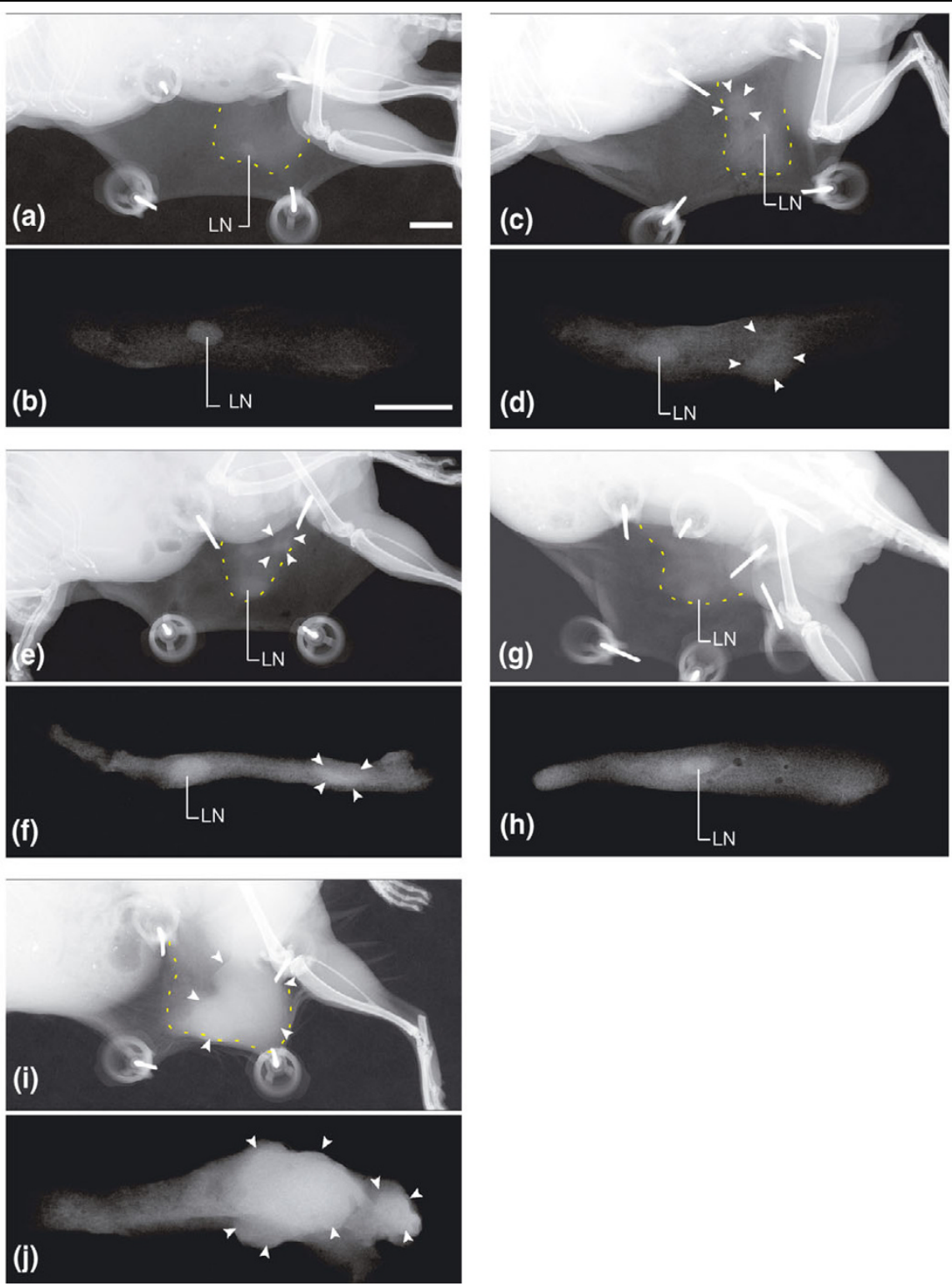

In vivo and ex vivo images of normal and altered mammographic density. Panels $\mathbf{a}, \mathbf{c}, \mathbf{e}, \mathbf{g}$ and $\mathbf{i}$ are mammographic images from live mice, and panels $\mathbf{b}, \mathbf{d}, \mathbf{f}, \mathbf{h}$ and $\mathbf{j}$ are the same glands imaged ex vivo. Mice were treated as follows: panels a and b, untreated mouse; panels $c$ and $d$, mammary gland inoculated with fibroblasts; panels e and $\mathrm{f}$, mammary gland injected with matrigel; and panels $\mathrm{g}$ and $\mathrm{h}$, day 14 of gestation. Panels I and $\mathrm{j}$ are from an MMTV-PyMT mouse with multiple mammary tumors. The size bar in all panels is $5 \mathrm{~mm}$. LN, lymph node.

\section{Conclusion}

This study establishes the mouse as a model for mammographic density research and demonstrates the feasibility of mouse mammographic imaging. Here we show for the first time that mouse mammography can be carried out in vivo. Furthermore, we demonstrate that mammographic density can be experimentally induced and imaged in a live mouse. Areas of density were generated experimentally through individual manipulation of stromal fibroblasts, epithelium, or matrix, which produced qualitatively distinct mammographic images both in vivo and ex vivo. Further- more, Pten-deficient mice and MMTV-PyMT transgenic mice served as genetic models in which to investigate whether the inherent structure of mammary gland correlates with mammographic density. The hypoplastic epithelium in Pten-deficient mice resulted in glands with low mammographic density and, conversely, hyperplastic glands of MMTV-PyMT exhibited pronounced radiographic density.

This new line of research faces many challenges. One of these is the need to maintain experimentally generated 
mammographic density over a time period that is sufficiently prolonged to allow development of the pathophysiological changes that lead to mammary cancer in mice. Comparisons between highly dense and less dense mammary glands may reveal differences in their susceptibility to mammary tumor formation. There is also a need to quantify and standardize mouse mammograms accurately, using means such as those developed for human mammography [26-28]. This will allow research on mammographic density using genetic mouse models that have either a loss-of-function or gain-of-function of genes linked to breast cancer. Specifically, it will help us to explore the relationship between genetic changes, mammographic density and breast cancer susceptibility. This work forms the basis for exploiting the mouse as a model in which to study mammographic density.

\section{Competing interests}

None declared.

\section{Acknowledgements}

The authors wish to thank Ms B Joseph for technical assistance, and Ms T Kalliomäki for providing the MMTV-PyMT mouse. Mmtv-cre mouse used to generate the Pten conditional knockout mice was kindly provided by Drs L Hennighausen and K-U Wagner. This research was supported by funding from the Canadian Breast Cancer Research Alliance to RK, and Margaret and Lee Chair of Breast Cancer Research to NFB. GAW is supported by a fellowship from the Susan G Komen Breast Cancer Foundation.

\section{References}

1. Johns PC, Yaffe MJ: X-ray characterisation of normal and neoplastic breast tissues. Phys Med Biol 1987, 32:675-695.

2. Ingleby HG-CJ: Comparative Anatomy, Pathology and Roentgenology of the Breast Philadelphia: University of Philadelphia Press; 1960.

3. Wolfe JN: Risk for breast cancer development determined by mammographic parenchymal pattern. Cancer 1976, 37:2486-2492.

4. Yaffe MJ, Boyd NF, Byng JW, Jong RA, Fishell E, Lockwood GA, Little LE, Tritchler DL: Breast cancer risk and measured mammographic density. Eur J Cancer Prev 1998, Suppl 1:S47-S55.

5. Boyd NF, Byng JW, Jong RA, Fishell EK, Little LE, Miller AB, Lockwood GA, Tritchler DL, Yaffe MJ: Quantitative classification of mammographic densities and breast cancer risk: results from the Canadian National Breast Screening Study. J Natl Cancer Inst 1995, 87:670-675.

6. Boyd NF, Jensen HM, Cooke G, Han HL, Lockwood GA, Miller AB: Mammographic densities and the prevalence and incidence of histological types of benign breast disease. Reference Pathologists of the Canadian National Breast Screening Study. Eur J Cancer Prev 2000, 9:15-24.

7. Brisson J, Merletti F, Sadowsky NL, Twaddle JA, Morrison AS, Cole $P$ : Mammographic features of the breast and breast cancer risk. Am J Epidemiol 1982, 115:428-437.

8. Buchholz TA, Wazer DE: Molecular biology and genetics of breast cancer development: a clinical perspective. Semin Radiat Oncol 2002, 12:285-295.

9. Byng JW, Yaffe MJ, Jong RA, Shumak RS, Lockwood GA, Tritchler $\mathrm{DL}$, Boyd NF: Analysis of mammographic density and breast cancer risk from digitized mammograms. Radiographics 1998, 18:1587-1598.

10. Byrne C, Schairer C, Wolfe J, Parekh N, Salane M, Brinton LA, Hoover R, Haile R: Mammographic features and breast cancer risk: effects with time, age, and menopause status. J Nat/ Cancer Inst 1995, 87:1622-1629.
11. Goodwin PJ, Boyd NF: Mammographic parenchymal pattern and breast cancer risk: a critical appraisal of the evidence. $A m$ $J$ Epidemiol 1988, 127:1097-1108.

12. Saftlas AF, Wolfe JN, Hoover RN, Brinton LA, Schairer C, Salane $\mathrm{M}$, Szklo M: Mammographic parenchymal patterns as indicators of breast cancer risk. Am J Epidemiol 1989, 129:518-526.

13. Wolfe JN, Saftlas AF, Salane M: Mammographic parenchymal patterns and quantitative evaluation of mammographic densities: a case-control study. AJR Am J Roentgenol 1987, 148:1087-1092.

14. Boyd NF, Lockwood GA, Byng JW, Tritchler DL, Yaffe MJ: Mammographic densities and breast cancer risk. Cancer Epidemiol Biomarkers Prev 1998, 7:1133-1144.

15. Alowami S, Troup S, Al-Haddad S, Kirkpatrick I, Watson PH: Mammographic density is related to stroma and stromal proteoglycan expression. Breast Cancer Res 2003, 5:R129-R135.

16. Guo YP, Martin LJ, Hanna W, Banerjee D, Miller N, Fishell E, Khokha R, Boyd NF: Growth factors and stromal matrix proteins associated with mammographic densities. Cancer Epidemiol Biomarkers Prev 2001, 10:243-248.

17. Warren R, Lakhani SR: Can the stroma provide the clue to the cellular basis for mammographic density? Breast Cancer Res 2003, 5:225-227.

18. Fata JE, Kong YY, Li J, Sasaki T, Irie-Sasaki J, Moorehead RA, Elliott R, Scully S, Voura EB, Lacey DL, Boyle WJ, Khokha R, Penninger JM: The osteoclast differentiation factor osteoprotegerinligand is essential for mammary gland development. Cell 2000, 103:41-50.

19. Fata JE, Leco KJ, Moorehead RA, Martin DC, Khokha R: Timp-1 is important for epithelial proliferation and branching morphogenesis during mouse mammary development. Dev Biol 1999 211:238-254.

20. Moorehead RA, Hojilla CV, De Belle I, Wood GA, Fata JE, Adamson ED, Watson KL, Edwards DR, Khokha R: IGF-II regulates PTEN expression in the mammary gland. J Biol Chem 2003, 278:50422-50427.

21. Wagner KU, Wall RJ, St-Onge L, Gruss P, Wynshaw-Boris A, Garrett $L$, Li M, Furth PA, Hennighausen L: Cre-mediated gene deletion in the mammary gland. Nucleic Acids Res 1997, 25:4323-4330.

22. Backman SA, Ghazarian D, So K, Sanchez O, Wagner KU, Hennighausen L, Suzuki A, Tsao MS, Chapman WB, Stambolic V, Mak TW: Early onset of neoplasia in the prostate and skin of mice with tissue-specific deletion of Pten. Proc Natl Acad Sci USA 2004, 101:1725-1730.

23. Borellini $\mathrm{F}, \mathrm{Oka} \mathrm{T}$ : Growth control and differentiation in mammary epithelial cells. Environ Health Perspect 1989, 80:85-99.

24. Richert MM, Schwertfeger KL, Ryder JW, Anderson SM: An atlas of mouse mammary gland development. J Mammary Gland Biol Neoplasia 2000, 5:227-241.

25. Sheffield LG: Organization and growth of mammary epithelia in the mammary gland fat pad. J Dairy Sci 1988, 71:2855-2874

26. Pawluczyk O, Augustine BJ, Yaffe MJ, Rico D, Yang J, Mawdsley GE, Boyd NF: A volumetric method for estimation of breast density on digitized screen-film mammograms. Med Phys 2003, 30:352-364

27. Shadagopan A, Alcorn FS, Semmlow JL, Ackerman LV: Computerized quantification of breast duct patterns. Radiology 1982 , 143:675-678

28. Saha PK, Udupa JK, Conant EF, Chakraborty DP, Sullivan D: Breast tissue density quantification via digitized mammograms. IEEE Trans Med Imaging 2001, 20:792-803. 